\title{
Challenges Faced During eCTD and CTD Filling Procedures for USFDA and Canada
}

\author{
Nisar Ahammad*, Nagarjuna Reddy, M.V. Nagabhushanam, Brahmaiah Ramakrishna \\ Department of Drug Regulatory Affairs, Hindu college of Pharmacy, Amaravathi road, Guntur-522006
}

\begin{abstract}
Electronic Common Technical Document (eCTD) is a topic of increasing interest in the pharmaceutical Industry as it become compulsory for filing procedures. The Common Technical Document (CTD) is a set of specification for application dossier, for the registration of Medicines and designed to be used across Europe, Japan and the United States.Quality, Safety and Efficacy information is assembled in a common format through CTD .The CTD is maintained by the International Conference on Harmonisation of Technical Requirements for Registration of Pharmaceuticals for Human Use (ICH). Electronic common technical documentis an interface used by applicants of marketing authorisation for medical products to submit regulatory affairs document to the agency concerned. The purpose of this article is to present a concise overview of challenges faced during eCTD \& CTD submissions in United States and Canada. A regulatory process, by which a person/organization/ sponsor/innovator gets authorization to launch a drug in the market, is known as registration process. The registration process will be done by submitting technical information to the authority
\end{abstract}

Keywords: electronic common technical document (ECTD)/ (CTD), International conference on hormonisation (ICH), Drug registration process.

Article Info: Received 11 June 2019; $\quad$ Review Completed 24 July 2019; $\quad$ Accepted 31 July 2019; Available online 15 August 2019

\section{Cite this article as}

Ahammad N, Reddy N, Nagabhushanam MV, Ramakrishna B, Challenges Faced During eCTD and CTD Filling Procedures for USFDA and Canada, Journal of Drug Delivery and Therapeutics. 2019; 9(4-s):673-679

http://dx.doi.org/10.22270/jddt.v9i4-s.3334

*Address for Correspondence:

Nisar Ahammad, Department of Drug Regulatory Affairs , Hindu college of Pharmacy , Amaravathi road , Guntur-522006

\section{Introduction:}

The concept of electronic regulatory submissions is not new, and has been evolving in America and Europe since the late 1980s. The Food and Drug Administration (FDA) and others has worked with electronic submissions for more than a decade CANDA -(Computer Aided New Drug Application), Initiated in 1985 by FDA in US.

According to FDA it is efficient and high-quality analysis of data. Unfortunately, the CANDA era led to a proliferation of unique and proprietary formats for CANDAs, most of which required a stand-alone desktop computer on the desk of each regulatory reviewer. A whole variety of strategies for CANDAs emerged, from simple to complex. Each CANDA required a reviewer to learn a new system for accessing the data, a daunting task in many cases that few reviewers had time for. There were no standards for the structure of a CANDA and no common software platform or file format for the data. The results were mixed, many reviewers and sponsors were delighted with the efficient review that CANDAs provided, but others were unwilling to train on and use multiple different systems, sometimes simultaneously.
The FDA soon called a halt to the unstructured CANDA era.But this was certainly not the end of the submission of electronic data.

DAMOS-Drug Application Methodology with Optical Storage; Initiated by European regulatory Europe in 1989.

SEDAMM - SoumissionElectronique de Dossiers d'Autorisation de Misesur le Marché; Initiated by France in 1993.

MERS- Multiagency Electronic Regulatory Submission Project; Initiated by USA, Newzea land, and Australia in 1994.

MANSEV - Market Authorisation by Network Submission and Evaluation; Initiated by UK, Denmark, France, Italy and EMEA in 1997.

In 1997, ICH M2 Expert Working Group (EWG) started working closely with M4 (CTD), the ICH guideline that presents the agreed upon common format for the preparation of a well-structured Common Technical Document for applications that will be submitted to regulatory authorities. 
Simultaneously the FDA revealed the beginnings of a new method of electronic submission. The increasing volume of NDAs and the need for expedited review caused by the 1992 Prescription Drug User Fee Act (PDUFA) initiatives demanded that the FDA develop an approach for the efficient review of electronic data. The FDA was looking for a way to deal with the accumulating volumes of paper in its file rooms and the logistical problem of distributing sections of regulatory submissions to appropriate reviewers. By means of a series of guidance documents, the agency intended to carefully define the structure and technology that was acceptable for electronic submissions. In this way, the FDA could ensure a consistent set of electronic submission documents and reviewers could be comfortable that any electronically submitted data would be viewable in a familiar format. As a result, in 2002, eNDA and eANDAGuidance issued by FDA. Shortly after the first guidance documents were issued, electronic submission of New Drug Application(NDA) and Abbreviated New Drug Application(ANDA) documents became an emerging standard for many pharmaceutical sponsors, eliminating the need for manual printing, duplication, pagination, and other processes.
A significant milestone was the adoption in 2003 of the ICH eCTD Guideline v3.0 on the electronic Common Technical Document (eCTD), which is the electronic counterpart of the Common Technical Document (CTD; a harmonized structure and format for regulatory submissions).

\section{Common Technical Document}

Common Technical Document for applications that will be submitted to regulatory authorities. A common format for the technical documentation will significantly reduce the time and resources needed to compile applications for registration of human pharmaceuticals and will ease thepreparation of electronic submissions. Regulatory reviews and communication with the applicant will be facilitated by a standard document of common elements. In addition, exchange of regulatory information between Regulatory Authorities will be simplified

The CTD is organised into five modules. Module 1 is region specific and Modules 2, 3, 4 and 5 are intended to be common for all regions. In July 2003, the CTD became the mandatory format for new drug applications in the EU and Japan, and the strongly recommended format of choice for NDAs submitted to the FDA, US.

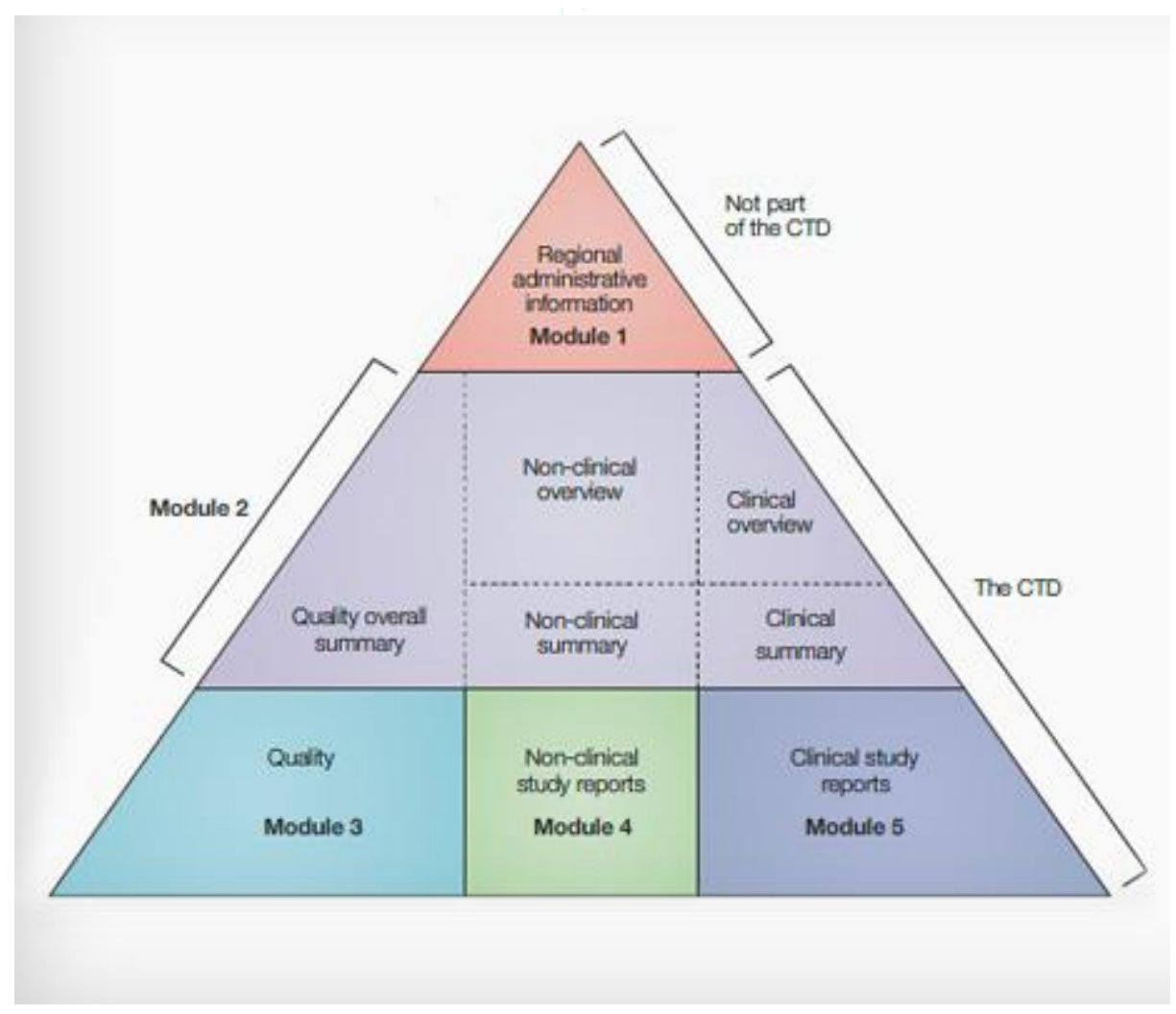

\section{eCTD}

eCTD or electronic common technical document is an interface designed for the pharmaceutical industry to transfer regulatory information. This module-based regulatory application format was developed by the International Conference on Harmonization (ICH M2 EWG). In 2008 the FDA (Food and Drug Administration) made eCTD format compulsory for all electronic submissions by FDA in 2008.

The life sciences industry has been facing certain difficulties in regulatory processes. Even though the electronic common technical document has been of great help in managing huge volumes of important documentation; there are a few glitches that complicate the submission process for the organizations. In this post we'll be discussing about those challenges faced by life sciences companies in eCTD submissions.

The role of eCTD is to help pharmaceutical companies enhance the submission procedure by bridging the gap between the time and market and minimizing expenses. However, a recent analysis made by the Open Text ${ }^{\mathrm{TM}}$ Corp (a leading company in enterprise content management), it's found out that most of the pharmaceutical companies are struggling hard to meet the deadline created by FDA to comply with the standard using eCTD format. 
eCTD format enables pharmaceutical companies to submit applications to various regulatory authorities such as FDA without altering the data. This XML-based electronic version of the CTD is now widely used as preferred submission format for the US Food and Drug Administration (FDA) and the European Medicines Agency (EMA).

eCTD format is now widely used in the United States, Japan, the European Union and Canada. As per Harv Martens, a member of the ICH M2 Expert Working Group,

"Pharmaceutical companies handle huge amounts of documentation for regulatory submissions. The information gathering process is very costly. It causes delays and expense in the development of new drugs. The objective of the CTD/eCTD is to harmonize both the content and the way it is delivered for the new drug approval process."

theeCTD is a standardized arrangement of documents that allows for the consistent and comprehensive presentation of information within a submission. eCTD submissions include 5 parts, termed modules, with each containing a specific type of information.

- $\quad$ Module 1 (not technically part of the CTD): regionspecific administrative information

- $\quad$ Module 2: manufacturing, nonclinical, and clinical overviews and summaries

- $\quad$ Module 3: detailed manufacturing information

- $\quad$ Module 4: nonclinical study reports

- $\quad$ Module 5: clinical study reports

\section{Chemistry, Manufacturing, and Controls (CMC)}

As anyone who has written an IND or marketing application knows, the CMC modules (module 3 and summaries in module 2) are expansive and can be tedious and timeconsuming to complete.

Because more documents typically mean more "cooks in the kitchen," it is tempting to combine permitted sections in an effort to streamline the management of authors and versions. However, the granularity of CMC sections should be written with the entire life cycle of the submission in mind.

For instance, if you decide to consolidate module 2.3 (Quality Overall Summary), any updates to drug substance sections will require the resubmission of this entire module rather than just the pertinent information.

As a general rule, a more granular structure should be considered in order to avoid issues throughout the lifecycle of the application. The "M4 Organization of the Common Technical Document for the Registration of Pharmaceuticals for Human Use" can be used as a reference to determine the permitted granularity.

\section{Clinical Study Reports}

Even experienced teams can encounter problems when determining the best way to structure a clinical study report (CSR) within the eCTD. Should it be submitted as a single document? If splitting it up, what is the proper way to group the sections?

The eCTD structure for Module 5 follows the levels outlined in "The Comprehensive Table of Contents Headings and Hierarchy." Although legacy CSRs can be submitted as one document, the current recommendation is to divide CSRs into sections: synopsis, report body, and individual appendices (i.e., Section 16 of the CSR separated to 3 rd level headings).

Preparing a CSR in this format allows reviewers to more easily navigate the large amount of information, leading to more efficient reviews. In addition, by dividing the report into sections, changes can be more easily tracked, as the entire report does not need to be replaced if only one section is updated.

\section{Report Numbering}

Every study report, both nonclinical and clinical, is required to have a study ID and title inserted into the submission. As such, it is important to ensure that all reports have a unique ID associated with them prior to sending to your publishing team. This typically becomes an issue when study data spans across multiple reports, such as an initial clinical study and associated population PK analyses. If a population PK analysis is being conducted for a specific clinical study, the population PK report should receive a unique study ID to ensure that the analysis is distinct from the general clinical study report. This also helps to ensure that any cross references in summary sections are clear as to the content being referenced.

\section{Annual Reports}

Back in paper days, the annual report was often written as a single document; however, the current granularity in "The Comprehensive Table of Contents Headings and Hierarchy" outlines multiple sections for annual reports.

While it may be tempting for sponsors to continue authoring the annual reports as one document, the structure of the eCTD does not easily allow for this approach and can lead to validation errors, putting the application at risk of a technical rejection.

If written as a single document, publishers may attempt to avoid validation issues by placing the annual report within a single node corresponding to one of the annual report sections (e.g., Summary for Nonclinical Studies). However, this approach risks confusion during the review, as the content will not properly align with the node description.

\section{Hyperlinking}

One of the most common issues that publishers encounter is attempting to hyperlink to a section and realizing that the section either does not exist or that it cannot be linked. Some of this is due to typographical errors during document authoring; however, many instances occur due to a lack of understanding by the authors of what content can actually be linked. As a general rule, hyperlinks can only be made to individual documents and not to section folders. For example, a frequent mistake when hyperlinking is referencing a section that is of a higher level of granularity than that to which the documents are written. Some examples include:

- Referencing the module for single dose toxicity studies (i.e., 4.2.3.1) rather than the specific study

- Referencing module 3.2.P.3 as a whole rather than the specific relevant section(s) (e.g., 3.2.P.3.1)

The "Comprehensive Table of Contents Headings and Hierarchy" and "M4 Organization of the Common Technical Document for the Registration of Pharmaceuticals for Human Use" can be used to determine permitted levels within an application.

The primary technical components are: 
- A high level folder structure (required)

- An EXtensible Markup Language (XML) "backbone" file which provides metadata about content files and lifecycle instructions for the receiving system.

- An optional lower level folder structure (recommended folder names are provided in respective modules of the eCTD specification below)

- Associated document type definitions (DTDs) and style sheets that support the presentation and navigation

\section{e-CTD ready document}

It is important that eCTD ready documents are prepared by authoring them in eCTD compliant templates. If this is not undertaken, a large amount of the "publishing time" is spent in document reformatting.

Guidance on the preparation of eCTD ready documents is provided below.

\section{a) File Organisation for the eCTD (Granularity)}

Refer ICH Topic M 4 Common Technical Document for the Registration of Pharmaceuticals for Human Use. Table 5 and Table 6 describe the levels in the eCTD hierarchy at which files should be placed and whether single or multiple documents are appropriate at each point. The tables describe Modules 2 and 3 with respect to the drug substance. For creation and maintenance of the files, the storage location does not have to be considered. The hierarchy structure will be applied during the compilation of the dossier.

\section{b) Specification for Submission Formats}

In general, documents that are provided in the different modules should be formatted as defined by the ICH Common Technical Document. Here it is described how files should be constructed for inclusion in the eCTD.Document) specification.

The eCTD submission is composed of the following:

\section{Directory structure}

- XML eCTD instance

- Content files

\section{- Directory structure}

The directory structure is a structure of directories and files. There should be a reasonable maximum number of entries (directories and files) per directory. The directory structure should follow the rules below. The files could be in several formats as specified below.

The name of the files and directories are identifiers. They should be short. The file names are not intended to convey meta-data, though some meaning in the name helps (i.e. no random names)

Recommended, but optional, names for directories and files are provided in appendix 4. Any directory names and file names that are added to the eCTD (Electronic Common Technical Document) submission by the applicant should be descriptive, logical and brief.

\section{XML eCTD instance}

The instance is in the submission sequence number directory. The submission sequence number directory should contain at least two files and one or more directories. One of the files in the submission sequence directory should be the instance and the other should be the MD5 checksum of the instance. The instance is the starting file for the processing by an XML (Extensible Markup Language) processor. The intention is to have links from the leaf elements of the instance to the files in the ECTD (Electronic Common Technical Document) submission as opposed to creating a single XML (Extensible Markup Language) document that contains the entire ECTD (Electronic Common Technical Document) submission. The instance also contains meta-data at the leaf level.

\section{eCTD template}

The ICH (International Conference on Harmonization) website (http://estri.ich.org/eCTD) includes an empty ECTD (Electronic common Technical Document) folder template as an example of an ECTD (Electronic Common Technical Document) submission folder structure. It shows all of the possible modules 2-5 folders as defined in appendix 4 and can be populated with the applicant data and edited as appropriate (i.e. adding additional folders or removing unnecessary folders). The applicant should still add the relevant regional module 1 folders and content, add the appropriate utility folders and content, and create the XML (Extensible Markup Language) index files to complete a valid ECTD (Electronic Common Technical Document) submission. The file formats included in this section are those formats that are commonly used in electronic submissions.

\section{File naming}

File names, including the extension, must not exceed 64 characters. Also folder names must not exceed 64 characters and the total file folder path length must not exceed 180 characters. Counting starts from the first digit of the sequence number in the sequence number folder name.

\section{PDF}

PDF is accepted as a standard for documents defined in this specification. Adobe Portable Document Format (PDF) is a published format created by Adobe. It is not necessary to use a product from Adobe or from any specific company to produce PDF documents. PDF is accepted as a standard for documents defined in this specification.

To ensure that PDF files can be accessed efficiently, PDF files should be no larger than 50 Megabytes. The files should be saved "optimized".

\section{Version}

Agencies should be able to read all PDF files with version 4.0 or higher of the Acrobat Reader. Agencies should not need any additional software to read and navigate the PDF files.

\section{Fonts}

Agencies cannot guarantee the availability of any fonts except Times New Roman, Arial and Courier and fonts supported in the Acrobat product set itself. Therefore, all additional fonts used in the PDF files should be embedded to ensure that those fonts would always be available to the reviewer. When embedding fonts, all characters for the font should be embedded, not just a subset of the fonts being used

The main role of eCTD in pharmaceutical industry is regulatory submissions. And it is done by harmonizing the blueprint and module-based format of pharmaceutical submission applications. This kind of submission ensures convenient and faster filing of applications. This interface is also upgraded by the ICH at regular intervals so as to make it more user friendly. 
For instance, in February 2015, ICH's M8 expert working group (EWG) released a draft implementation guide for ICH eCTD v4.0. It was the first major version change after the release of v3.0 in 2003.

\section{Advantages using eCTD}

- eCTD is built on acknowledged standards that haven't changed much in all these years in integrating ICH requirements

- Regulatory tools used to review submissions have been upgraded and hence offer sturdy performance

- It follows a common format for both US \& Europe with relatively simple changes ( Module 1 and STF acceptance)

- The life cycle offers detailed submission history alongwith easy knowledge transfer for product

- Consolidated formation offers transparency to submissions -

- Simple tools are used for -Publishing Submissions

- Method is quite similar to paper work

- Share the updates with multiple Local affiliates involved in the submission processes

- Viewer is not required during the submission process

- Affordable implementation

\section{eCTD challenges}

As eCTD format has become mandatory in the key markets, it has become essential for the companies to conceive a unified environment that aids the lifecycle of every kind of inherent submission. In this way, they can effortlessly manage the exhaustive system of accumulating, acclaiming, releasing and documenting new drug/medical device applications.

\section{Other eCTD challenges}

- $\quad$ Requires cutting edge technology and seasoned staff

- Not all the content is in a standard format. Hence standardization is required

- Regional differences seen in hyperlinking, bookmarking and PDF version

- Difficult to implement last minute changes

- Product knowledge is lost if the concerned employee quits

- Local affiliates have limited access to create or customize

- validation rules vary from region to region

- Baseline submissions are costly and likely to be of limited value

- Life-cycle management is challenging

- Differences seen in authentication of different regions

- Consolidated approach to drafting of dossier

\section{Challenges faced by life sciences companies in eCTD submissions}

In spite of all the benefits offered by the standardized framework of eCTD, there are still some setbacks with this kind of submission format that makes it quite a challenge for the life sciences companies. The major challenge is to find the regulatory tools that support the software adhering to the eCTD submission requisites.

In order to successfully carry out the eCTD submission, pharmaceutical companies must grasp all documents, including assortments, responses to questions, modifications and restorations in a consistent electronic format.

The pharmaceutical companies should use a primary software product to manage the integrated regulatory data and regulatory submissions process.

\section{Risks involved in eCTD publishing}

As the move from paper-based to eCTD submissions continues around the world, a multitude ofchallenges faces regulatory departments. But there are simple steps you can take to avoid common problems, which at best can increase the cost of or cause delays to your submission's approval, and at worst result in receipt of a Refusal To File.

Your submission publishing might be conducted by a dedicated, in-house department located in the same office or on the other side of the globe, or you might utilize thirdparty service providers. Your publishers might be highly experienced regulatory consultants with chemistry degrees, or specialized staff with administrative, IT or creative backgrounds. Whatever the case, busy publishing teams typically encounter the following 10 problems. Find out what you can do to avoid these problems and prevent or at least mitigate the risks of your eCTD publishing project.

\section{Source document incompatibility}

Today's electronic publishing software greatly speeds up the publishing process by scanning source documents to automatically extract information to use as navigational aids in the published output. In this process, which differs among file types (Word, PDF, etc.) and tools from different vendors, source files are scanned and elements such as internal document links, existing bookmarks and heading/outline styles are processed and collected into the software's database to create bookmarks and hyperlinks in the published output. If source files are not set up as the publishing software expects them to be, this process can be impaired and extra time may be required post publishing to manually add navigational elements. In companieswhere the whole submission preparation process (stats, medical writing, regulatory affairs, publishing, quality control, etc.) is conducted in house, setting up strict procedures and templates ensures the success of this process. However, if any of these functions is conducted externally, challenges increase and it is worth considering the following tips:

- Set up and use standard procedures, templates and forms, and distribute these to any externalservice providers.

- Publishing departments/providers should document and distribute the specifications andexpectations for source files to the concerned parties.

- Always ensure your source files are tested in the publishing software well before final publishing is scheduled. 


\section{Insufficient or conflicting information for publishers}

Depending upon the experience of your regulatory affairs and publishing staff and the lines of responsibility between them, critical information required in the publishing process might be unclear or ambiguous to publishers even though it is included in the content of your submission. It is prudent to provide all expected information to the publisher, however obvious this information may seem.By way of example, eCTD submissions rely heavily on the use of metadata, which provide additionalinformation about elements. In some cases, these metadata are included in critical capacities such as folder paths in the final eCTD. Providing this information to publishers at the same time as the source files using welldesigned procedures and forms is an easy way to prevent potential rework. It is fairly safe to say that ambiguity is the publisher's biggest enemy. If information is missing, progress is usually halted while the information is sought. However, if information is provided, but is ambiguous or conflicting, there is a real risk of the publisher's interpreting the information incorrectly and the error may not be discovered until too late, requiring major rework.

\section{Incorrect document versions}

From a publisher's perspective, there is nothing more souldestroying than working for days (or weeks) to complete publishing of a submission only to be informed that a wrong document or document version has been used. Unfortunately, all too often this means not only a large amount of rework but also the loss of full confidence in the integrity of the published submission, requiring moreintensive QC reviews. Publishing groups that utilize closed document management systems (DMS) in their publishing workflows generally avoid this problem because only those documents and/or versions marked as approved are available for publishing. Groups that use file shares for publishing repositories are more susceptible to this type of problem and therefore require far more stringent procedures.

\section{Short publishing timelines}

Submission publishing usually occurs at the end of a very long process. Time lost in previous stages of the process often is expected to be recovered during publishing. This poses little problem to those with access to large publishing departments or providers that can simply add more resources to reduce the time required on critical path. In smaller publishing operations where add- ing extra resources is not possible, aggressive timelines usually result in stressed publishers who are far more likely to produce error-laden submissions. It is sensible to allow extra time not only for the possibility of delay but also for other contingencies such as illness and problems with legacy files.

However, one of the most effective ways of mitigating risks to publishing timelines is to operate an incremental build policy, where modules or sections of your submission are published independently.Some parts of a submission normally are available for publishing weeks or even months before final publishing is scheduled to begin, and any possibility of publishing these sections outside the critical path will help adhere to the target time line.

\section{Nonlinear delays}

Not only are delays sometimes inevitable, (although they can be planned for, and in some cases mitigated), but they also can result in non- linear effects on the submission timeline.
For example, a delay of one or two days can be carried though the project and, if extra resources cannot be utilized, will result in a sub- mission that is one or two days late. But in other cases, especially where third-party providers are involved, delays of just a day or two may result in far more serious consequences. If the slot for publishing the project cannot be moved back by even a day or two due to conflicts with other scheduled projects, the one- or two-day delay may end up becoming a one- or twoweek (or worse) delay.

\section{Inappropriate granularity}

It has often been said that that eCTD publish-ing begins with the author because a document produced using a quality template with the appropriate level of granularity has such a huge effect on publishing. If you plan to submit a section as multiple leaves, these leaves should be supplied as the corresponding number of source documents rather than being rolled up into a single file for splitting during publishing. Every source document that must be sent back for reformatting is another small opportunity for the project to be delayed.

\section{Technical problems with legacy files}

Because some information may be produced many years prior to inclusion in a submission using outdated software and equipment, many opportunities exist for errors to surface during publishing. Although legacy files may have been printed without issue in the past, electronic publishing is extremely efficient in highlighting technical issues, often at the most critical time. These issues are generally not difficult to resolve, although they can be very time-consuming. Here, the most important tool in the publisher's toolbox is time, and by publishing submissions using incremental builds, these problems can be addressed well before they have opportunity to cause a delay.

\section{Quality Control reviewing at the right point}

By the time publishing begins, source file con- tent should be final and approved, as changing a document during the publishing process can have a devastating effect on the project timeline. Set clear QC points throughout the project but ensure those points are appropriate to the task:

- All source documents should be quality checked before entering the publishing workflow.

- The submission structure (the assembly/outline) within the publishing software should be independently reviewed prior to publishing.

- All published PDF files should be reviewed on screen.

- Check bookmarks and links in published PDF files.

- Always validate and conformity-check eCTD submissions prior to submission.

- Independently check all submission media and packaging prior to sealing and dispatch.

\section{Inappropriate validation process}

One of the real advantages of the eCTD is the ability to check its technical conformity upon submission. This means that both the applicant and the agency can be sure - from a technical perspective that the eCTD conforms to the specifications of the guide- lines under which it is being submitted. Conformity can be determined within days, or even hours, of being submitted, rather than the weeks or sometimes months required with paper submissions.

But this process has another advantage. Although the eCTD is considered an open standard and can, in theory, be 
produced and viewed using software from any vendor, in most cases the actual software used by the agency is also available to the applicant. This means that prior to submitting your eCTD to, say, the European Medicines Agency, you can validate it using the same software the agency uses (EursValidator) and view the same conformity reports on which it bases acceptance of the submission. As long as the electronic transfer of the files to the agency does not introduce any corruptions, you can be $100 \%$ confident that your submission will be acceptable (from a technical perspective) to the agency.

\section{References}

1. www.ivowen.com/regulatory-affairs-common-abbreviationsacronyms/
2. www.regulatoryinfo.org/challenges-faced-by-life-sciencescompanies-in-ectd-submissions/ www.regulatoryone.com/2011/10/ctd.html

3. ICH M2 EWG - eCTD specifications ICH M2 EWG Electronic Common Technical Document Specification version 3.2.2." (PDF). ICH. Retrieved 29 October 2015.

4. "Providing Regulatory Submissions in Electronic Format Certain Human PharmaceuticalProduct Applications and Related Submissions Using the eCTD Specifications" (PDF). US FDA.

5. www.fda.gov/RegulatoryInformation/Guidances/ucm126959 htm 